\title{
The medical microbiology teaching path
}

\section{Editorial}

The first time I attended to a master class of Microbiology at the Medicine School, I discovered a whole new world of amazing knowledge, almost magical, that made me fall in love for the rest of my days with the reign of the microscopic creatures. This romance with Microbiology increases not only in the course of the lessons of my second year of Medicine studies, but becomes greater when I start taking daily contact with the patients at the hospital, when I advance in other knowledge fields such as immunology, physiology, physiopathology, medical nosography, pediatrics, pharmacology, infectious diseases, tropical medicine, internal medicine, dermatology, gynecology, surgery, public health and epidemiology, and many others, that involves the subjects of study of the microbiology (bacteria, fungus, parasites, viruses, algae and even proteins -like prions-) in this body of knowledge.

My peers in other Medicine Schools hated Microbiology, why? Because for future Doctors the path to transmit microbiological knowledge is a real torture! The emphasis in taxonomical aspects, molecular structures, biochemical reactions, detailed life-cycles for every bug, etc., far away from the role that microorganisms plays in the disease production and how to understand it to then know how to suspect, how to perform a diagnostic (microbiological demonstration) and at last, how to treat it and prevent it. I was so lucky, because my professors understood that a medicine student, a future doctor, needs specialized concepts in "medical" microbiology, not the huge background of knowledge that involve microbiological sciences, so, at my Medicine School (Jose Maria Vargas Med School, Medicine Faculty, Universidad Central de Venezuela), a new way to teach microbiology began, a path to follow the pathogenicity of the microorganism and its interactions with the human host (susceptible) to produce a specific disease, in different organs and systems, from hands of one great human being and professor, Dr. Felix Oswaldo Carmona Guillod, and the rest of the teaching group of medical microbiologist specialist that he led and trained in this successful path of bringing high quality scientific complex knowledge in a pleasant and useful way to the students.

I try to illustrate this particular mode of teaching microbiological science focused on medicine students: I take a lower respiratory tract infection as an example to show it: let's begin with the microorganism and its "entrance" to the susceptible host, I'm talking of the pneumococci, Streptococcus pneumoniae, a Gram-positive cocci, in pairs or couples, I always remember the words "a romantic cocci" (because the diplococci disposition "front-to-front" resemble a couple kissing, or the flames of two candles); surrounded by a capsule (one of its major pathogenicity determinants, that protects them against opsonization, phagocytosis and mediates adherence -specific and not; and become in target for the diagnostic test -as Quellung reaction-, or even vaccines directed against the capsular polysaccharide), that colonize the pharynx of the host evading protective mechanism like mucosal surface immunoglobulin, with adherence to specific receptors of the upper respiratory tract epithelium, producing inflammation that modify the quality of the mucous fluid, interfere with the movement of the ciliary epithelium (mucociliary clearance), stimulates a nonefficacy cough reflex, that finally leads to the access to the lower

\author{
Volume 7 Issue 2 - 2019
}

\author{
Marcel Marcano-Lozada, ${ }^{1,2}$ Maria Valentina \\ Marcano-Sanabria, ${ }^{3}$ Maria Valeria Marcano- \\ Sanabria ${ }^{3}$ \\ 'Aggregate Professor Microbiology Department, “J.M.Vargas" \\ School of Medicine, Universidad Central de Venezuela, Venezuela \\ ${ }^{2}$ Medical Microbiology Specialist, Medical Microbiology Unit, \\ Angios Vascular Center \& Wound Clinic, Venezuela \\ ${ }^{3}$ Escuela Normal Superior General José de San Martin, Carlos \\ Casares, Argentina
}

Correspondence: Marcel Marcano-Lozada,Aggregate Professor Microbiology Department, "J.M.Vargas" School of Medicine, Universidad Central de Venezuela, Caracas, Venezuela, Tel +506-57190516, Email marcelmarcano@gmail.com

Received: February 20, 2019 | Published: March 04, 2019

respiratory airways of the microorganism, and its "fight" against the alveolus defense mechanism (surfactant, alveolar macrophages), concludes in the establishment of an inflammatory reactions that let the white blood cells gain access to the alveolus lumen (pneumococci chemotactic factors "calls" the immune cells), and then the production of the degradation of these cells result in the presence of anexudate that blocks the exchange among non-oxygenated blood and the oxygenated air that can't reach the terminal alveolus, and as a terminal point induces a necrotic process of specific parts of the respiratory tract (with a classic radiological image known as pulmonary atelectasis).

Nowadays I only talk about pathogenicity, with a little bit of immunology, because the med student needs to suspect the possible etiological agent of this disease, how it does? Medical microbiology teaching for future doctors doesn't need to explain the composition of each polysaccharide of the capsule, its interest is focused first in lead the student to think what is the possible microorganism responsible for the signs \& symptoms of the pathology, and this approach comes based on epidemiological concepts, like the knowledge of the most frequent pathogens for age-group (newborn, scholar, adolescent, adult, elderly), for condition \& risk factors (smokers, diabetic, HIV, etc.), provenance (community or healthcare setting), according to therapeutic history (previous consumption of antibiotics in the last 3-6 months for example), vaccination record, contacts (relatives with the same disease, students at the same school with similar symptoms, etc.), and with a good interrogation in the making of the clinical record, the orientation completes with the physical exam findings and laboratory tests results. At this point, medical microbiology reveals its real significance for the future doctor, it is a discipline that provides the knowledge to confirm the suspicious of the healthcare practitioner, and the opposite of many people's belief, that microbiology performs diagnosis.

The real importance comes walking the diagnostic path. It begins with the sample selection to perform a good sample collection, for example, in the previous example, sputum is the optimal sample because it contains viable microorganism, that can be revealed by stains \& culture, but the rules for the collection of the best $\&$ 
representative sample should be observed (first morning sputum, without mouth hygiene, low quantity of saliva, a high densitysample -mucous thick-, and with some microscopic characteristic like more than 25 polymorphonuclear cells by observation field \& low than 10 epithelial cells). Then, the ideal sample collector (light protection, wide, sterile), the adequate conservation (at room temperature, noted refrigeration), the quick transport to the microbiology laboratory, and a very, very important part of the diagnostic confirmation process: FULL INFORMATION (patient ID information, age, sex, contact info from patient $\&$ physician, sample type, date/hour collection, epidemiological \& clinical relevant data, pharmacological record, current treatment, type of microbiological studies to be perform -stains, culture, PoC test, serological, etc.-), to ensure the adequate interpretation of the microbiological findings and its quickly availability to the healthcare personnel. In this specific case, performing of blood culture is an option, but, nor bacteremia information to support this procedure was mention, so, its indication could be a discussion point.

And finally, the susceptibility test results. Many physicians wait for it to adjust antimicrobial therapy, but the medical microbiology teaching wants only a fine-tuning in cases when the clinical evolution of the patient is not the best, so, if you suspect and corroborate a bacterial agent, your treatment goes directly to it from the first moment, and the antibiogram is a support tool to help other aspects like the switch therapy for example. The difference among medical \& clinical microbiology approaches comes with the critical interpretation of the findings of stains, isolates, identification and susceptibility tests, because in the medical approach, the results supports (or not) a therapeutic decision, and could be refuted or suspicious to be a false negative or positive according to the patient background and the clinical setting. All the previous highlights made me fall in love with medical microbiology teaching and then practice (in the microbiology laboratory and the clinical setting -external consultation, hospitalization ward, emergency room, etc.), with the possibility to improve sample collection, clinical data recording, transportation, suggestion of specialized test or PoC methods to support a quick management, and then, from the laboratory, interact and teach my colleagues (physicians) about the right importance of the microbiological resources.

The knowledge of the etiology (microorganism) and the pathogenesis (mechanism to survive and harm host), leads to know how to diagnose the disease agent (microbiological demonstration) and how to treat them (susceptibility and antimicrobial therapy), without the need to become an expert in the number of flagella or a guru that remembers how many porins has an specific microorganism, in order to bring the patient the most accurate attention to restore (or preserve) their health. So, I consider a key factor for the future physicians' success, that acquire medical microbiological knowledge in the early stages of their professional formation, before getting to the hospitalization ward, and previous to the complete pathophysiology and therapeutics subjects, to ensure the optimal articulation of all the parts of the knowledge to assemble the infectious disease that affects the patients.

Another important consideration, medical microbiology requires physicians training future physicians (or specialist), not only biologists or microbiologists, virologist, bacteriologist, mycologists or parasitologists; medical microbiology approach needs physicians with a specific formation in the lab \& clinical settings, and with an special training in teaching techniques, to bring the adequate amount of complexity and useful information to the future doctors, because, microbiological details are fascinating, but, many of them aren't necessary to the diagnosis \& treatment of an infected patient. It is important to develop a laboratory training, not pretending that every future physician becomes an expert when a Gram stain is in the microscope, instead, the medical microbiology approach pursuit that the future physician understands what is in the microscope, the basic staining, morphology and aggrupation, and made a link between its suspicious and the objective observation findings; further, identification of the description of a colony type that grows in a specific plate, or, generates a doubt or think in an mechanism of resistance to an antimicrobial agent when a susceptibility test is placed in front of it.

Medical microbiology teaching approach on etiopathogenesis and microbiological diagnose is a great, practical \& useful approach to bring the tools to the healthcare professionals to fight against the world of the microscopical enemies, and after more that 25 years of experience, I'm sure that this approach works, because many of my students gave me the right feedback from the academic \& the clinical setting, and still reminds the knowledge that I bring to them in our medical microbiology lectures and laboratory sessions. So, I'll never forget the first phrase at my initial microbiology master class "Welcome to the world of the microscopic", because that welcome made me know an incredible small world that helped me become a better physician for my patients through the microbiological knowledge.

\section{Acknowledgments}

None.

\section{Conflicts of interest}

Authors declare that there is no conflicts of interest. 